\title{
Nanoparticles visualization and extraction for diagnosis and therapy in nanomedicine
}

\author{
Adham Aleid ${ }^{1 *}$, Khalid Alhussaini ${ }^{1}$, and Ali S. Saad ${ }^{1}$ \\ ${ }^{1}$ Dept. of Biomedical Technology, King Saud University, Riyadh 11433, Saudi Arabia
}

\begin{abstract}
Nanomedicine is a rapidly developing field of science that has the potential to treat a wide range of complicated ailments. This paper uses a mouse with an inflamed calf and iron oxide nanoparticles (IONPs) attached to the therapeutic medicine and put into the mouse's eye to investigate drug delivery efficiency. The idea is to track and quantify drug delivered to the inflamed calf of the mouse. A highresolution MRI approach was used to capture images of the inflammatory calf area. Knowing that iron oxide has a high magnetic strength in MRI, image processing techniques were used to calculate the position and number of IO-NPs linked to the administered medication. This paper proposes an image processing approach for detecting and extracting IO-NPs. The images go through pre-processing steps that includes noise filtering and background removal. IO-NPs are isolated from the surrounding tissues using Otsu's method. The number of IO-NPs grouped in the region, as well as the quantity of medications supplied to the region of interest, can be estimated using IO-NPs extraction. The findings on nanoparticle detection and extraction appear to be a potential method for estimating the amount of medicine targeting a specific location.
\end{abstract}

\section{Introduction}

When complicated image processing tasks, such as spatiotemporal tracking, counting, and classifying huge numbers of cells, are required, computerized viewing and monitoring of biological cells in multiple imaging modalities is becoming increasingly critical. Cell recognition and spatiotemporal tracking have become popular in recent years [1]. However, because it needs the use of specialized computer procedures, it is a difficult task to complete. Threshold approaches are employed to define cell outlines, and they are frequently sophisticated, owing to the inherent problem of inadequate contrast between cells and their surrounds [2]. To boost contrast and identify cells from tissues, cells must be injected with a contrast agent.

Molecular magnetic resonance imaging can be utilized to monitor cells in vivo and noninvasively if the contrast agent possesses magnetic characteristics . The use of iron oxide (superparamagnetic iron-oxide (SPIO)) for in vivo monitoring of biological processes at cellular level is a relatively novel technology. MRI, hyperthermia treatment [3], tissue healing [4], and drug delivery systems (DDSs) are all examples of biological applications where NPs exhibit unique features. Because of their biocompatibility, acceptable surface design, and simplicity of conjugation with targeted ligands, SPIOs are employed as intracellular contrast agents.

MRI has become one of the most extensively utilized imaging modalities for in vivo monitoring of numerous cell types since it is non-invasive, produces highresolution images, and does not rely on radioactive isotopes [5]. For diagnosis and treatment purposes, the ability to visualize the three-dimensional (3D) distribution of SPIO is crucial. However, automatic and precise quantification of the amount of SPIO-labeled cells, as well as display of the 3D data, are required.

The distribution of high-resolution MRI images in the region of interest remains a difficult problem to solve [6].

\section{Method}

A new image processing technique for 3D visualization and quantification of IO-NP labeled macrophages is proposed in this study, which is based on high-resolution MR images of inflammation-bearing mice calf muscle. Pre-processing of the MR images is used to improve contrast and reduce noise. Data is segmented using an automatic threshold algorithm. To observe the 3D distribution of NPs and increase the accuracy of detecting the number of IO-NP tagged cells in the region of interest, a method for collecting IO-NP particles was also created as described below.

\subsection{Image processing and quantification}

The following sections show how to identify, detect, and visualize SPIO labeled macrophages in the mouse calf. First, from the whole collection of photos encompassing the field of interest (e.g., 30-50 images), a sub-stack was chosen in the region of interest (inflammatory site).

\footnotetext{
* Corresponding author: adaleid@ksu.edu.sa
} 


\subsection{Background subtraction}

Context subtraction is commonly used in motion detection in films. All techniques for moving objects, such as the temporal median filter, Mixture of Gaussians, Kernel density estimation (KDE), and, Sequential KD approximation, are insufficient for our data because we have still images. On each of the 50 images in a stack, context subtraction was performed using the identical processing parameters. The "Rolling Ball" algorithm was selected because it removes smooth continuous backgrounds from images, which is ideal for MRI images [7]. The local background value for each pixel is calculated by averaging across a somewhat large ball around it. The "Rolling Ball" radius in 8-bit or RGB images should be at least as large as the radius of the image's largest nonbackground object. Unless the image's context is overly skewed, larger values are also appropriate. For 16-bit images with a wide range of pixel values, standard radius values are set between 1 and 5 pixels.

\subsection{Contrast enhancement}

The IO-NPs were reversed in the stack of images to look as white dots or clusters of dots, which improved the visual quality. To better differentiate the NPs from other objects in the image, the stack was contrast improved using the histogram equalization approach.

\subsection{Segmentation}

A standardization technique was performed to make sure that all pixel values were scaled to be between 0 and 1 . This technique can be applied to photographs taken from various fields. Each image in the stack undergoes the normalizing procedure then the values are scaled to be in the range $[0,255]$. Automatic local threshold segmentation is the next stage, which is appropriate for the processed data because it removes the bias of manual selection and is completely independent of imaging parameters [8]. Over 17 automatic threshold approaches in ImageJ were tested as part of the pre-selection investigation. To choose the best segmentation, a comparison with a control group of photos was performed. In the control set of images, there were no NPs. The image's local histogram was used in the automatic threshold technique [9]. It was chosen to detect the NPs in the image because it delivered the least number of noise particles in the control images [10].

\subsection{Morphological operations}

After segmentation, the image was reduced to a binary format with only two levels: black background and white objects, which included the sought NPs. Morphological techniques on the binary image were essential to clear all the outlying pixels that were considered noise. An opening of a two-pixel radius erosion followed by a dilation of the same radius was used to maintain the image's structure and dimension. To expose only NPs in the ensuing stack of images, the leg structures in images, such as bone and muscles, were eliminated.
ImageJ's "Analyze Particles" method was employed, with parameters set to produce images with only the linear structure and no NPs, assuming that NP or aggregation of it does not form linear shapes. Non-particles, on the other hand, might be spherical or elliptical in shape, and are referred to as NPs. To limit the appearance of this instance, we set up the settings of the particle analysis process using a control stack that did not include NPs as a reference picture. By subtracting the input image from the particle analysis's output image, the image of NPs distribution was obtained.

\subsection{Quantification of NPs}

Finally, the size of NPs or an agglomeration of them was assessed using white dots with numerous pixels. A control stack without NPs was utilized instead of other images containing NPs. Particle analysis was used to automatically count the average number of particles in the stack of photos with 3D visualization.

\section{Results}

The method was tested using MRI imaging equipment on a 3D calf model, although processing was done on each 2D image; each 3D model included roughly 50 images. Figure 1a shows the image with the SPIO designated macrophages in white after background subtraction and grey level inversion.

The threshold in Figure 1b was used to segment the image in Figure 1a. The image of the structure of the mouse calf in binary format can be seen. Figure 1c displays a depiction of the leg structure without NPs. The "Particle Analyze" tool in ImageJ was used to extract the spherical and elliptical small particles and construct the mouse calf structure (Fig. 1b). By subtracting the pictures Fig. $1 \mathrm{~b}$ from the Fig. 1c, the SPIO labeled macrophages were obtained in the image in Fig. 1d, which depicts their distribution in a slice of the inflamed region. This was done using ImageJ's "Analyze Particles" technique, set to yield images with only linear structure and no NPs. It was assumed that NP, or aggregates of it, did not form linear shapes. While technically plausible, occurrence likelihood was extremely low, hence it was overlooked. NPs, on the other hand, are non-particles that can take on spherical or elliptical geometries. To reduce risk, a control stack of MRI images of the same area was used to pick the parameters of the "particle analysis" procedure.

The average number (percentage) of circular and elliptical shapes left in the reference stack after applying the "particle analysis" approach to the reference stack was calculated to be roughly $1 \%$ of the overall average number of counted NPs per slice. A 3D view of the complete leg is provided by the green circle ringing the region where the spherical forms reflect the SPIO NPs in Fig. 2a. Multiple spherical forms reflecting the $3 \mathrm{D}$ positions of the NPs marked by yellow arrowheads, which are encompassed by a yellow rectangle in Fig. 2a, may be seen in Fig. $2 b$. 

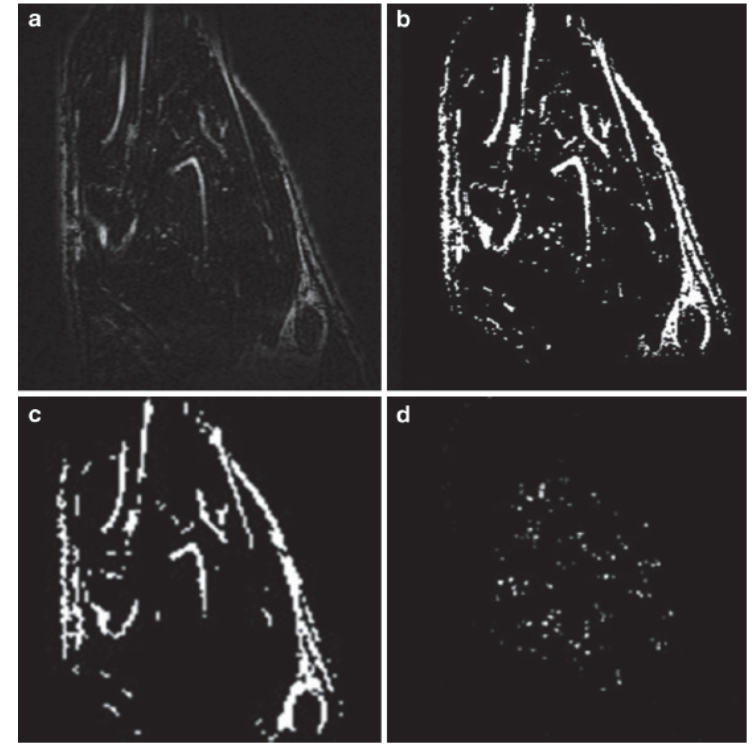

Fig. 1 a) Image of the mouse calf after background subtraction and inversion of the grey levels. b) After segmentation, c) without nanoparticles and d) 2D distribution of SPIO labeled macrophages inside the region of interest in a single slice 3D Visualization of iron oxide nanoparticles.

\section{Discussion}

Our method is the first to use non-invasive highresolution 3D MR imaging to view and semiautomatically detects SPIO labelled macrophages within an infected mouse calf, allowing us to determine the volume of the inflamed location and detect macrophage homing for more accurate diagnosis. It's also simple to count the amount of IO-NPs in the calf. Counting the voxels in the $3 \mathrm{D}$ cube of data can reveal the number of voxels occupied with IO-NPs. Before estimating the number of IO-NPs within the inflamed region, which is proportional to the number of blue voxels, we need to know how many single SPIO-NPs can fit in a voxel. This method of 3D visualization of NPs will surely benefit nanomedicine researchers, particularly in the field of cancer, in optimizing their diagnosis and treatment strategies by assuring drug delivery to the tumor utilizing an MRI imaging device.

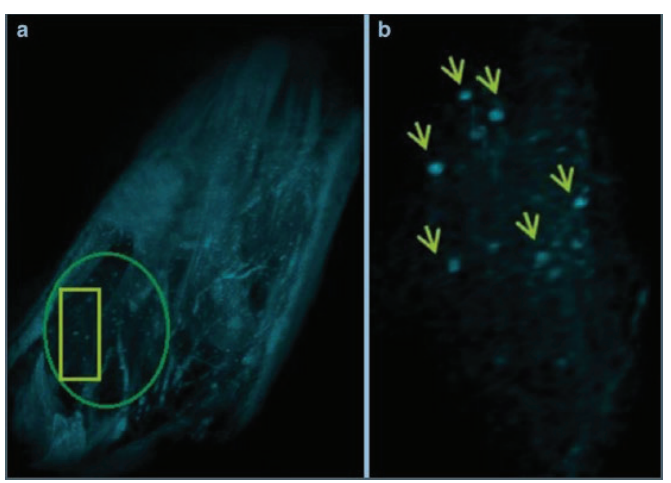

Fig. 2 a) Shows a 3D view of the whole leg, the green circle surrounding the area where the spherical shapes represent the SPIO NPs and $b$ is a zoom of the area surrounded by yellow rectangle in a.

\section{Conclusion}

The suggested method demonstrated promising potential in evaluating in vivo, targeted drug delivery. It was able to identify SPIO-labeled macrophages from mouse calf tissues and visualizes iron oxide NPs in three dimensions. In a variety of nanomedicine applications, the ability to visualize the 3D distribution of NPs inside the region of interest aids in the diagnosis and treatment of targeted NPs.

\section{References}

1. A. Al Faraj, N. Luciani, J. Kolosnjaj-Tabi, E. Mattar, O. Clement, C. Wilhelm, F. Contrast Media Mol Imaging, Gazeau, Real-time high-resolution magnetic resonance tracking of macrophage subpopulations in a murine inflammation model: a pilot study with a commercially available cryogenic probe. 8 (2), 193-203 (2013)

2. A. Arbab, J. Frank, Regenerative Med, Cellular MRI and its role in stem cell therapy. 3 (2):199-215, (2008)

3. J. Bulte, D. Kraitchman D, NMR Biomed, Iron oxide MR contrast agents for molecular and cellular imaging. 17 (7):484-499, (2004)

4. S. Berman, P. Walczak, J. Bulte, Wiley Interdiscip Rev Nanomed Nanobiotechnol, Tracking stem cells using magnetic nanoparticles. 3 (4):343-355, (2011)

5. A. Gupta, R. Naregalkar, V. Vaidya, M. Gupta, Nanomedicine, Recent advances on surface engineering of magnetic iron oxide nanoparticles and their biomedical applications. 2 (1):23-39, (2007)

6. H. Mok, M. Zhang, Expert Opin Drug Deliv, Superparamagnetic iron oxide nanoparticle-based delivery systems for biotherapeutics. 10(1):73-87, (2013)

7. K. Li, M. Chen, T. Kanade, Med Image Comput Comput Assist Interv, Cell population tracking and lineage construction with spatiotemporal context. 10(Pt 2):295-302, (2007)

8. W. Liu, J. Frank, Eur J Radiol, Detection and quantification of magnetically labeled cells by cellular MRI. 70(2):258-264, (2009)

9. S. Telrandhe, A. Pimpalkar, Kendhe, World Conference on Futuristic Trends in Research and Innovation for Social Welfare (Startup Conclave), Detection of brain tumor from MRI images by using segmentation \& SVM. (pp. 1-6). IEEE., (2016)

10. S. Iqbal, J. Sheetlani, Biotechnology Research Asia, Application of modified $\mathrm{K}$ means clustering algorithm in segmentation of medical images of brain tumour. Biosciences. 14(2), 735-739., (2017) 